\title{
Did the Human Genetic Lineage Start with Comet Impacts at the End of the Eocene?
}

\author{
N. Chandra Wickramasinghe $e^{1,2,3,4}$, Gensuke Tokoro $^{2,4}$ and Maximiliano C.L. Rocca ${ }^{5}$ \\ ${ }^{1}$ Buckingham Centre for Astrobiology, University of Buckingham, UK \\ ${ }^{2}$ Centre for Astrobiology, University of Ruhuna, Matara, Sri Lanka \\ ${ }^{3}$ National Institute of Fundamental Studies, Kandy, Sri Lanka \\ ${ }^{4}$ Institute for the Study of Panspermia and Astroeconomics, Gifu, Japan \\ ${ }^{5}$ Mendoza 2779-16A, Ciudad de Buenos Aires, Argentina \\ Email: ncwick@gmail.com
}

\begin{abstract}
The theory of cometary panspermia posits that the evolution of life on Earth including the introductions of novel innovative trends is controlled to a large extent by the incidence of bacteria and viruses from the wider cosmos. In this communication we point out the possibility that the defining characteristics of H. sapiens - intelligence, acuity of vision amongst others - may have been introduced into ancestral Anthropoidea primates 35-40 million years ago (Mya) when the Earth was being showered with cometary debris.
\end{abstract}

Keywords: comets, primates, Eocene, human evolution

\section{Introduction}

The theory of cometary panspermia and the concept of a cosmic biology argues that the evolution of life on a planet like the Earth cannot be regarded as confined to that planet. Viruses and bacteria have a cosmic origin with the development of life on a planet involving the piecing together of these genome fragments against environmental selection criteria together with the operation of Lamarckian-Darwinian selection processes. Horizontal gene transfer (HGT) that has been empirically verified at a terrestrial level over the past 3 decades, would in our view, have its fullest scope for expression on a grand cosmic scale. The phenomenon of punctuated equilibrium highlighted by Gould and Eldredge (1977) is a manifestation of the stochastic nature of the interaction of the solar system with a galaxywide distribution of gene-carrying dust, comets and planetary bodies.

\section{Solar System in Relation to the Galaxy}

Our solar system orbits around the centre of the galaxy with a period of $\sim 240 \mathrm{My}$, whilst also oscillating in the z-direction (about the galactic mid-plane) with a half period of $\sim 40$ years (Stothers, 1998, 2000). It could be argued that each galactic plane crossing could trigger a flux of comets from the Oort cloud into the inner solar system leading to enhanced rates of collision of cometary bodies with the Earth. Particularly intense showers of comet impacts are furthermore expected to occur whenever the solar system passes through one of the spiral arms (Napier and Clube, 1979). These episodes would be linked to clusters of cratering events and to periods of mass extinctions of species. Mass extinctions of species such as the Permian extinction (252Mya) and the Cretacious-Tertiary extinction (65Mya) could be explained by impacts arising when the solar system crossed the Scutum-Crux and Sagittarius-Carina arms respectively (Leitch and Vasisht, 1998). The process of large-scale extinction of species is thought to arise from a combination of direct impact destruction of phyla and habitats combined with accompanying climatic perturbations. An additional important factor could be the introduction of disease-causing agents (pathogens) from the new cometary flux.

On the cometary panspermia hypothesis, comets that contain viruses and bacteria interact with evolving biology on the planet to cause emergence of new species as well as modifications to existing phyla. While the bulk of gene additions that have occurred over the past 4.2 billion years contributing to the slow cumulative evolutionary changes are expected to have occurred via comets that are 
indigenous to the solar system, comets from outside our solar system can bring in novel suites of genes on a sporadic basis. The great Cambrian explosion of life, for instance, may be explained by such a process of gene additions probably involving ingress of extra-solar comets during close encounters of our solar system with extrasolar planetary/cometary systems (Rocca and Acevedo, 2014).

Within the solar system as many as $30 \%$ of all long-period comets that approach perihelion actually leave the solar system in hyperbolic orbits. Comets in initially bound elliptical orbits $(\mathrm{e}<1)$ could be deflected into hyperbolic orbits due to the effect of gravitational perturbation by Jupiter. Because the solar system thus produces a steady exodus of comets the principle of mediocrity requires other similar planetary systems, of which there are now known to be hundreds of billions, to do likewise. Due to technical difficulties only recently have two candidate exocomets in hyperbolic orbits been observed Oumuamua which reached perihelion on 9 September 2017, and Borisov which reached perihelion on 8 December 2019 (Wickramasinghe, et al, 2018).

\section{History of Life on Earth}

Bell et al (2015) recently discovered the oldest evidence of microbial life on Earth in rocks dated older than 4.1Bya which are now exposed in the Jack Hills outcrop in Western Australia. The evidence is in the form of graphite microspheres (residues of bacteria) within zircon crystals, with the ${ }^{12} \mathrm{C}$ enhancement relative to ${ }^{13} \mathrm{C}$ signifying biological origin. The fact that this represents the earliest evidence of terrestrial microbial life occurring during the Hadean geological epoch effectively removes the possibility of any primordial soup on the Earth. The impact-riddled Hadean epoch clearly points to impacting comets as the natural deliverers of this microbial life.

The subsequent history of terrestrial life is dictated by three main factors: (a) geological processes leading to an evolution of the Earth's crust and atmosphere and consequently to variations and changes of habitats; (b) impacts such as the K/T impactor causing extinctions of species; and (c) comet dusting leading to the introduction of new bacteria and viruses leading in turn to gene transfers over a galactic scale. It is against this backdrop that the evolution of terrestrial life occurs, significantly in fits and starts and including protracted episodes of stasis or relative quiescence.

\section{Human Beginnings?}

In the last phase of the geological epoch known as the Eocene 38-34 Mya there is now clear evidence of an enhanced flux of cometary dust indicated by ${ }^{3} \mathrm{He}$ and Ir anomalies associated with chondritic spinels, as well as of large impact craters (Boschi et al., 2017, Farley et al, 1998). There are at least five large impact craters/structures on Earth with radiometric ages placed around 35 million years ago (Osinski \& Pierazzo, 2013). They are:

1) Popigai in Russia, (100 km in diameter)

2) Chesapeake in USA, (90 km)

3) Toms Canyon submarine offshore in USA, (22 km)

4) Mistastin Lake in Canada, $(16 \mathrm{~km})$

5) Wanapetei in Canada, $(8 \mathrm{~km})$

This time also signals the end of a 250 million-year greenhouse epoch and the beginning of the present so-called "icehouse" condition of our planet. From Fig 1 we note that this period also coincides remarkably with a surge of the Haplorhini suborder of primates leading eventually to modern humans (Williams et al, 2010; Gingerich, 2012). Jaeger et al (2010) further reported that the high taxonomic diversity of anthropoids at Dur At-Talah in central Lybia indicates a rapid colonization of Africa by multiple anthropoid clades at some time during the middle Eocene epoch, coinciding with the comet dusting episode. A similar report by Gebo et al (2012) shows that an analogous situation occurs in regard to species of Eocene Primates from Shanghuang, China at precisely the same period.

In conclusion our speculation would be that our own distinctive genetic heritage leading to Homo sapiens, which sets us apart from all other lifeforms on the planet, was somehow acquired in the protracted shower of viruses that would have accompanied the cometary dust injected to Earth via impacts during the closing stages of the Eocene epoch some 35-38 million years ago. This is shown in 
the divergences radiating out of the blue circle of Figure 1 (Macdonald et al (2001)) at precisely this time.

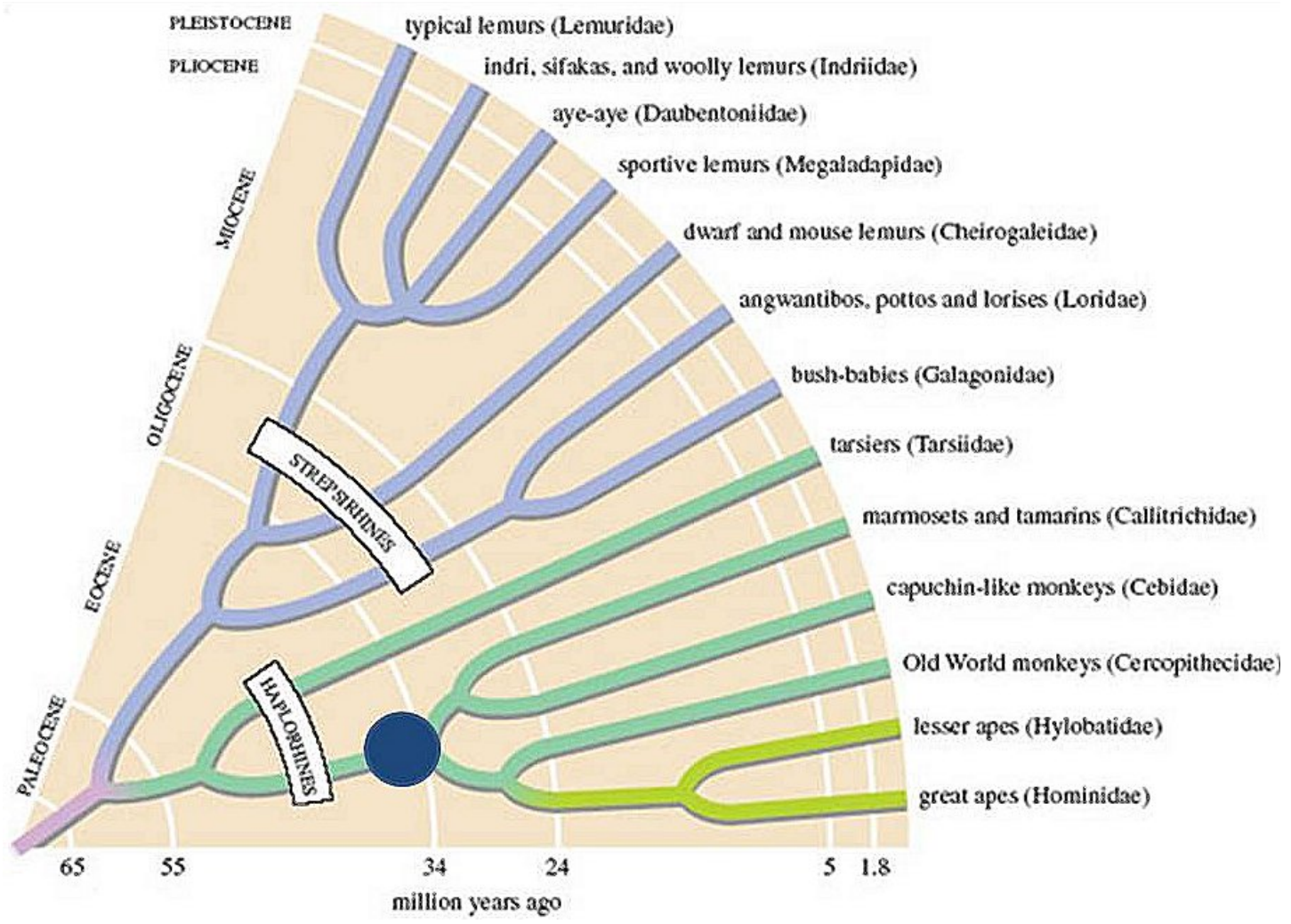

Figure 1. Diagram showing primate lineages beginning predominantly in the mid to late Eocene. These findings have been largely vindicated in later finds of fossils both in Africa and China, modified and updated from a chart published in the New Encyclopeadia of Mammals in 2001.

\section{References}

1. Bell EA, Boehnke P, Harrison T et al (2015). Potentially biogenic carbon preserved in a 4.1 billion-year-old zircon, PNAS, 112 (47) 14518-14521 www.pnas.org/cgi/doi/10.1073/pnas.1517557112

2. Boschi, S., Schmitz, B., Heck, P.R. et al, 2017. Late Eocene 3He and Ir anomalies associated with ordinary chondritic spinels, Geocheim. Et Cosmochim. Acta, 204, 205-218

3. Farley, K.A., Montanari, A., E.M., Shoemaker,E.M. and Shoemaker, C.S., 1998., Geochemical evidence for a comet shower in the late Eocene, Science, 1250-1253

4. Gebo, D.L., Dagosto, M., Ni, Xijun et al, 2012. Species Diversity and Postcranial Anatomy of Eocene Primates from Shanghuang, Chin, Evolutionary Anthropology 21:224-238

5. Gingerich, P.D., 2012. in Messell and the terrestrial Eocene - Proceedings of the $22^{\text {nd }}$ Senckenberg Conference, Palaeobio Palaeoenv, DOI 10.1007/s12549-012-0093-5

6. Gould, S. J. and Eldredge, N., 1977. Punctuated equilibria: the tempo and mode of evolution reconsidered, Paleobiology 3 (2): 115-151

7. Hoyle, F. and Wickramasinghe, N.C., 1981. in C. Ponnamperuma (ed) Comets and the Origin of Life, p.227, (Dordrect; D.Riedel)

8. Jaeger, J., Beard, K., Chaimanee, Y. et al. 2010. Late middle Eocene epoch of Libya yields earliest known radiation of African anthropoids. Nature 467, 1095-1098

9. Leitch, E.M., and Vasisht, G., 1998. New Astron, 3, 51

10. Macdonald, D. (ed.) 2001. The New Encyclopedia of Mammals ( Oxford University Press)

11. Napier, W.M. and Clube, S.V.M., 1979. Nature, 282, 455 
12. Osinski, G. and Pierazzo, E., (editors), 2013: Impact Cratering: Processes and Products. Wiley Press, Chichester, $\mathrm{UK}, 330 \mathrm{pp}$.

13. Rocca, M.C.L. and Acevedo, R.D., 2014. Extrasolar comets in our solar system captured during close encounters with nearby stars? Abstract 5170 presented at the 77th Annual Meeting of the Meteoritical Society.

14. Stothers, R.B., 1998, Mon.Not.Roy.Astr.Soc., 300, 1098

15. Stothers, R.B., 2006, Mon.Not.Roy.Astr.Soc., 365, 178

16. Wickramasinghe, J., Wickramasinghe, C., Napier, W., 2010. Comets and the Origin of Life (World Scientific Publ.)

17. Wickramasinghe, N.C., Steele, E.J., Wallis, D.H. et al, 2018, Advances in Astrophysics, Vol. 3, No. 1,

18. Williams, B.A., Kaya, R.F. and Kirkb, E.C., 2010. New perspectives on anthropoid origins, PNAS, 107(11), 4797-4804 